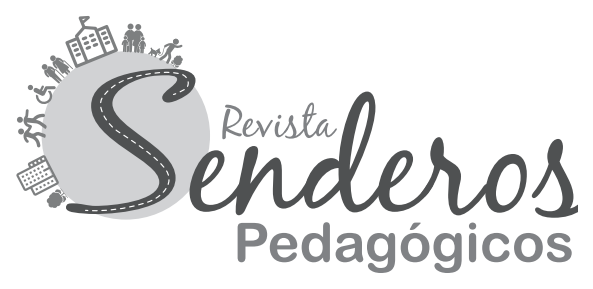

\title{
La configuración de la identidad nacional, el currículo y la enseñanza de la literatura ${ }^{1}$
}

\author{
The configuration of national identity, curriculum and teaching \\ of literature
}

\author{
Autor: \\ Juan Carlos Hernández Palencia² \\ Recibido: 30/06/2020 \\ Aprobado: $03 / 11 / 2020$
}

1. Artículo de reflexión.

2. Doctor en Letras de la Universidad de Buenos Aires, Magíster en Literatura Colombiana de la Universidad de Antioquia, Especialista en Currículum y Prácticas Escolares en Contexto de la Facultad Latinoamericana de Ciencias Sociales (FLACSO) de Argentina. Investigador del Grupo de Estudios Interdisciplinarios sobre Educación (ESINED) de la Universidad de San Buenaventura Medellín. jhernandezpalencia@yahoo.fr

\section{Resumen:}

En el presente artículo se propone la reflexión sobre algunos paradigmas sociales y culturales que se configuran en el currículo académico, a partir de dos elementos que componen sus discursos cotidianos: el sentido de realidad y la noción de convivencia. Para ello se recurre al lenguaje que se emplea en su delineamiento y ejecución articuladamente con los contextos y las circunstancias ideológicas que lo intervienen, y desde los cuales se configura la idea de identidad nacional, a partir de posiciones críticas con las cuales se cuestionan y sugieren direcciones de cambio, en aras de contribuir a condiciones sociales y humanas diferentes.

Palabras clave: investigación pedagógica, investigación sobre el currículo, ambiente educacional. 


\begin{abstract}
:
This article proposes the reflection on some social and cultural paradigms that are configured in the academic curriculum from two elements that compose their daily discourses: the sense of reality and the notion of coexistence. This is done by using the language used in its delineation and its execution in articulation with the contexts and ideological circumstances
\end{abstract}

that intervene in it, and from which the idea of national identity is configured, based on critical positions with which they question and suggest directions of change, in order to contribute to different social and human conditions.

Keywords: pedagogical research, research on the curriculum, educational environment.

\section{Introducción}

Este ejercicio se proyecta como una propuesta en tanto que, habiendo realizado una lectura analítica de carácter socio crítico y hermenéutico a los documentos de carácter oficial y/o privado, cuestiona y re significa las propuestas curriculares; por lo tanto, implicará analizar enunciados que contengan formas ideológicas, las cuales se configurarán en las acciones que desde allí se direccionan como documentos rectores, en los cuales "un movimiento del pensamiento va del todo a las partes y de las partes al todo", de modo que las partes reciben significado del todo y todo adquiere el sentido de las partes.

Este artículo es además uno de los resultados de la investigación documental $^{3}$ - financiada por la Universidad de San Buenaventura durante el 2019- y que está vinculada con otras investigaciones y productos inscritos en la misma línea de reflexión y pensamiento en las que se viene trabajando hace varios años ${ }^{4}$.

Los currículos y demás documentos revisados son en su gran mayoría de carácter público, y sirven como directriz a las instituciones educativas, ya que en ellos se estipulan formas, métodos y contenidos; en algunas ocasiones también son de carácter privado, pues corresponderán a las instituciones educativas en las cuales no operaría, de manera total y directa, el discurso ministerial.

En concordancia con lo anterior, se realiza una lectura transversal de estos elementos, con el ánimo de establecer las relaciones entre la configuración de identidad nacional, el currículo y la enseñanza de la literatura. A la vez, se pretende potenciar una propuesta curricular que resignifique el proceso de enseñanza-

3. Hacia la configuración de identidad nacional a través de la enseñanza de la literatura en América latina. Acta de inicio código M5856, Facultad de Educación, Universidad de San Buenaventura Medellín.

4. Ver: “Aportes para una antropología de la pedagogía latinoamericana”. Pacarina del Sur [En línea], año 11, núm. 43, abriljunio, 2020. Congreso Internacional de la Cátedra UNESCO para el mejoramiento de la calidad y equidad en la educación con base en la escritura y la lectura para América Latina. (2018). Universidad de Cuenca, Ecuador. 
aprendizaje de la literatura, como configuradora de ciudadanías e identidades, atendiendo a circunstancias especificas, más que a lineamientos estatales de carácter estandarizante, pues cuestionar las nociones de currículo, de los procesos de lectura como acciones que configuran comportamientos en los lectores en tanto que legitiman - a partir de los actos discursivos que emplean — las nociones de realidad, moralidad y ética que, a su vez, reconfiguran o conservan esas estructuras sociales.

Para empezar5 ${ }^{5}$, se comprenderá el currículo, sobre el cual tienen injerencia las políticas educativas, como lo define Grundy (1998):

El currículum, no es un concepto, sino una construcción cultural. Es decir, no se trata de un concepto abstracto que tenga algún tipo de existencia fuera y previamente a la experiencia humana; más bien es un modo de organizar una serie de prácticas educativas (p. 19).

Por lo tanto, es una práctica tan compleja, sobre la que no es extraño encontrarse con perspectivas diversas que seleccionan puntos de vista, aspectos parciales, enfoques alternativos con distinta amplitud que determinan la visión “más pedagógica” del currículo.

Desde allí, y en relación con las reflexiones y planteamientos orientados hacia la descripción y caracterización del currículo con los temas que lo transitan, Andrea Brito y Silvia Finocchio (2006) han realizado varias reflexiones en relación a este asunto en concreto, las cuales han planteado la conformación de algunas disciplinas escolares. Desde su perspectiva, los currículos de América Latina se han centrado en los saberes elementales que forman parte de la alfabetización inicial: la historia, la geografía, las ciencias sociales, la matemática y, últimamente, en las nuevas tecnologías de la comunicación y la información.

Aquí es posible comprender que, no solo las transformaciones de la sociedad contemporánea imponen nuevos desafíos a la educación; la rica y compleja historia de los saberes transmitidos por la escuela invitan también a una reflexión. En este sentido, se desarrollan algunas consideraciones que se espera contribuyan a abrir la clásica pregunta sobre qué enseñar y que enriquezcan las iniciativas que hacen de la escuela un espacio de construcción cultural.

La concepción y puesta en práctica de un currículo escolar pretende, en primera instancia, definir los objetos de estudio pertinentes, las formas pedagógicas y didácticas adecuadas con las cuales ejecutarlo como proyecto educacional; y en segunda instancia, suministrar y velar por el constante aprendizaje de los valores

5. La primera parte de este artículo es, en gran medida, parte del trabajo de grado de la Especialización en Currículum y Prácticas Escolares en Contexto, FLACSO, Argentina, con el nombre de El currículo escolar o el lenguaje vacío (2013); texto que aún no ha sido publicado. 
morales y sociales que promueve, con el fin de hacer que los individuos en proceso de educación se inserten de la mejor manera posible en la sociedad y la cultura en la que coexisten, ya que el currículo “[...] cumple una función social clave, la de proyectar y articular elementos tanto provenientes de la herencia cultural como surgidos recientemente, y esbozar un contorno nuevo de realidad y sociedad" (De Alba, 2007, p. 3). En otras palabras, la educación es la forma práctica y menos traumática de incorporar al individuo en la cultura y la sociedad que lo contienen, con el fin de hacerlo un sujeto que las desarrolle y las conserve.

En consecuencia, el currículo, en estrecha relación con el imaginario de realidad desde el que es construido, ayuda a que este imaginario sea conservado, reproducido, expandido o contraído, según se haga necesario; para ello, el lenguaje con el que se configura se hace fundamental, ya que articula de manera consistente la construcción, delimitación y modificación del sujeto en tanto que este habita en lo simbólico.

En esa medida, la carga significativa con la que se ha llenado este lenguaje encuentra su origen en la concepción occidental de cultura, desde la que fueron configurados y determinados los "valores", las "virtudes" y, en oposición, los “defectos" y "anomalías" con las que se definió y diferenció —casi de manera automática- el bien del mal, lo bello de lo feo y lo valioso de lo sin valía; generando con ello una brecha entre los que "poseen la cultura" y los "bárbaros"; donde el sujeto culturizado occidentalmente fue establecido como única forma posible del Ser, al considerar que las relaciones que pudieran establecerse entre el sujeto (quien conoce y domina) y el objeto (el mundo, la naturaleza, el otro que es conocido y dominado) pudieran darse solo en esa dirección, lo cual entronizó una concepción irreductible del Ser para vincularse con el mundo y la realidad, la del dóminus y con ello, la idea "indiscutible" de que esa forma relacional es única y natural.

Esta concepción determinista del sujeto en la cultura occidental ha conllevado hacia aparatosas y contradictorias formas la existencia del hombre contemporáneo, pues todo aquel entorno natural del que debía enseñorearse y beneficiarse, ahora se agota por el abuso; y todo aquel que considerara bárbaro, carente de cultura -el otro, el ajeno simplemente- es educado igualmente con la idea de competir, de "ser mejor que ...” de "tener más que ...” de "llegar más lejos que ...”, y de ser necesario, matar simbólica o físicamente, para saberse así “mejor que ...”; poniendo en entredicho los grandes paradigmas de la historia con los que se ha querido promover lo contrario: liberté, égalité, fraternité.

Desde esta perspectiva, estos elementos al entrar en tensión han dado lugar a dos paradojas que se manifiestan, particularmente, en el lenguaje del currículo. 


\title{
Primera paradoja: el lenguaje en contradicción con la noción de realidad
}

\author{
[... la sociedad no está a la par de lo que se hace en el colegio, la \\ sociedad no responde a lo que estamos enseñándoles. Nosotros les \\ decimos que hay que estudiar, que esforzarse y eso a la sociedad no \\ le interesa (Kessler, 2002, p. 38).
}

De maneras semejantes a lo planteado en esta referencia, las operaciones entre el lenguaje con el que se construye y con el que opera el currículo y los sujetos que transitan por él, aparecerán como actos de comunicación fallidos que irán vaciando de sentido todo discurso promotor de actitudes y comportamientos necesarios para la cualificación del proceso educativo; ya que el lenguaje con el que pretende promoverse actitudes y comportamientos sociales éticos, aparece desvirtuado en tanto que encuentra en el sentido de "realidad" un vacío en el que es imposible materializar su sentido, y el cual parece no ser capaz de configurar y devolverle, siquiera, un eco; donde su sentido parece quedar vulnerado ante la ligereza con la que se asimila dentro y fuera de los ámbitos escolares.

Visto de otra forma, las inconsistencias que se presentan entre los discursos sociales con los cuales pretenden configurarse aquellos parámetros ideológicos que conlleven hacia formas "civilizadas", "racionales" — por ende "generadoras" de bienestares socialmente instituidos- y los obtenidos en los ámbitos de la realidad cotidiana, entran en conflicto al no corresponderse con el acto que debería identificarlos. El comentario hecho por un estudiante: "Ya era muy obvio, cuando te das cuenta de que los profesores no van nunca. La hora empieza 7 y 20 de la noche y bajabas y la profesora estaba tomando mate" (Kessler, 2002) es una prueba de ello. Las interpretaciones de este ejemplo son diversas, y muchas harán hincapié en la subjetividad y la particularidad de la misma, o apuntan hacia lo muy "desubicada" que está la maestra; sin embargo, es en estos actos de realidad - ya sean ejecutados por profesores, por padres, por las estructuras sociales que configuran la idea de mundo en general-, en los que el lenguaje no encuentra un sentido y no logra configurarse como vínculo entre la sociedad, la cultura, el currículo y el sujeto de la comunidad.

Así los "valores” y "virtudes" instituidos, además de generar una primera contradicción al ser modelos rectores concebidos en concordancia con la verticalidad e imponencia del sujeto determinista de la cultura occidental - a los cuales se hizo referencia anteriormente-, encuentran una segunda contradicción en la que las inconsecuencias entre lenguaje y acto se hacen cada vez más evidentes, y donde conceptos como: respeto, lealtad y responsabilidad empleados para dar seguridad, apoyo, sentido de pertenencia e igualdad para educar, pasan a ser enunciados carentes de sentido que no significan nada, ni siquiera algo que pueda definirse, mucho menos materializarse en actos de convivencia. 
- ¿Sabes qué es el respeto?

-Sí claro, es respetarse y respetar al otro, donde vos no te metes en la vida de nadie y nadie se mete con vos [...] no meterse en la vida de nadie $[. .$.$] (Definición realizada por un joven de 15$ años).

\title{
Segunda paradoja: la generalización de la cultura curricular y la particularización de la cotidianidad
}

\begin{abstract}
Los profesores consideran que los alumnos tienen 'una conciencia muy clara de que hay que saber, pero no saben para qué hay que saber', y en los años superiores se expresan cuestionamientos del tipo 'para qué tengo que saber si puedo llegar a cosas sin saber', a lo que los profesores tienen dificultad de responder, sobre todo porque secretamente comparten la duda (Kessler, 2002, p. 38).
\end{abstract}

De manera más coloquial, pero en el fondo el mismo interrogante se lo plantea un agricultor que va a una escuela rural a continuar con su proceso educativo: "iserá que aprender inglés me sirve para algo?”.

Los conceptos y los referentes establecidos como parámetros globales de realidad -el saber y el conocimiento en los casos referidos- que se insertan y convalidan, entre otras cosas, a partir del currículo, parecen entrar en contradicción con los que se establecen como formas de lo particular, pues no encuentran una correspondencia ni una concordancia entre aquello concebido como ideal (el deber ser) y la realidad en tanto inmediatez (lo que se es), generando un tipo de "corto circuito", en el que terminan divorciados el acto y su sentido. Es decir, las formas determinantes que provienen en gran medida del pensamiento y la cultura occidental, imponen formas concretas de Ser, en tanto que:

[...] se nos presenta como una sucesión de triunfos: el de la verdadera religión sobre los paganismos, el de la razón sobre la tradición, el del conocimiento sobre la ignorancia o el de la tecnología sobre las limitaciones intrínsecas de la vida terrenal (Navarrete Linares, 2001).

Esto contribuye en gran medida a desvirtuar el sentido de la educación y del aprendizaje, a la par que expone en alto grado los demás componentes inherentes al mismo proceso (responsabilidad, trabajo, cooperación, etc.), ya que de alguna manera el discurso que se elabora desde el currículo para generar y proveer certezas y bienestares al sujeto susceptible de educación, se ficcionaliza; pues aparece ante "otra realidad" que se le opone, lo delimita y aquello que debería implicar su posesión 
y apoyo, se queda en la ficcionalidad discursiva del currículo que, evidentemente, no podrá suministrar nada de lo dicho.

De esta manera, la realidad cotidiana del sujeto "su real y única realidad" se convierte por oposición y descarte en su única forma posible de ser y existir, sin necesidad de que asuma de manera consciente una postura subversiva o siquiera crítica, respecto a lo que lo circunda. Su comportamiento tenderá hacia el subsistir antes que hacia el convivir. Dicho de otra manera, el sujeto que habita en la negación de sí dentro de la cultura construye otras formas posibles de ser, en las que la negación de toda forma diferente a la propia, siendo esta su forma posible de ser, sea la única forma de ser.

En El malestar de la cultura, Sigmund Freud comenta sobre el amor humano por el prójimo como forma posible de generar y mantener la convivencia:

[...] todos los esfuerzos de la cultura destinados a imponerlo aún no han logrado gran cosa. Aquélla espera poder evitar los peores despliegues de la fuerza bruta concediéndose a sí misma el derecho de ejercer a su vez la fuerza frente a los delincuentes; pero la ley no alcanza las manifestaciones más discretas y sutiles de la agresividad humana (1930, p. 41).

En esta observación, se entrevé que los intentos realizados por la sociedad y la cultura a partir de la educación resultan infructuosos en tanto que intenta combatir un comportamiento que ella misma promueve al considerarlo "natural", pues más adelante en el mismo texto dice:

Sin embargo, sería injusto reprochar a la cultura el que pretenda excluir la lucha y la competencia de las actividades humanas. Esos factores seguramente son imprescindibles; pero la rivalidad no significa necesariamente hostilidad: sólo se abusa de ella para justificar ésta (1930, p. 41).

Así, al considerar como natural toda forma de competencia que se justifique en el desarrollo de la humanidad, en el comportamiento estulto ${ }^{6}$ del sujeto moderno, el cual está más empeñado en el hecho de que la humanidad crezca en complejidades y susceptibilidades asociadas a la idea de falso progreso y falsa comodidad, en vez de estarlo en cosas que impliquen aumento de bienestar, de moralidad, de dominio del mundo.

6. La estulticia que concibe Michael Foucault (1994) refiere dos sentidos: el primero, la apertura a las influencias del mundo exterior, recepción absolutamente acrítica. El segundo, la dispersión en el tiempo que deja que su vida discurra sin más, es decir, el que no dirige su voluntad hacia ningún fin. 
Toda forma posible para generar actitudes diferentes a las mencionadas no podrán encontrar mayor resonancia, y solo sería posible en tanto los paradigmas que han sido establecidos como ejes fundamentales del currículo y del prospecto occidental, respecto a las formas posibles del Ser, sean modificados; de lo contrario, continuará siendo evidente la inaplicabilidad e impertinencia de un currículo que ahonde en la cuidadosa elaboración de la teoría curricular y la muy descuidada circunstancialidad de los sujetos sobre los que pretende operar.

El problema de lo pedagógico en América Latina, supone bajo el principio de la incomprensión y la incapacidad del hombre latinoamericano para asimilar adecuadamente su propia totalidad, su propia realidad a la luz de sus propias circunstancias y por ende querer encontrarla en la insuficiencia de elementos, fórmulas, y métodos ajenos; lo que ha implicado un salir de sí, para, como en un reflejo, encontrar en las reglas y en las teorizaciones que dan cuenta de otra realidad y por ende de otra esencialidad, una base que le permita "comprender" y teorizar — por homologación de leyesla suya propia, a través de la ajena, aunque de igual manera, no las comprenda; llegando incluso, a modificar drásticamente la realidad simbólica y geográfica en al que habita, para que la teoría importada se articule en ella pretendiendo explicarla, pero a ciencia cierta no llega siquiera a describirla (Hernández Palencia, 2020).

De la contextualización anterior puede comprenderse que la forma como se plantean las propuestas curriculares y pedagógicas están en profunda relación con las formas ideológicas que caracterizan a la sociedad y a la cultura en la que son pensadas o quizás, realmente, en como son imaginadas.

Bajo ese principio, los lineamientos curriculares que se presentan como directrices ministeriales para tal ejercicio, se configuran como puntos referenciales que pretenden unificar los procesos, pero esto no garantiza que puedan ser tomados como modelos o propuestas realmente ejecutables debido a las variables y a las circunstancias heterogéneas en las que pretende ejercerse una propuesta con carácter homogenizante.

Las directrices diseñadas y planteadas como formas determinantes en los procesos de enseñanza aprendizaje están directamente articuladas con los materiales de carácter pedagógico y proyectadas a lograr un grado de acoplamiento y dinamismo con las planeaciones realizadas por los profesores, pero esperando siempre que en esa articulación se logre un funcionamiento específico que garantice el alcance de dichos logros en los procesos. El asunto es que dichos derroteros son formas adscritas a una condición determinada bajo ciertos parámetros, pero sobre todo a ciertos intereses 
que consideran las formas de bienestar, como unas que están relacionadas con formas incongruentes de realidad, en donde el bienestar está orientado hacia la posesión de ciertas cosas, y no hacia el mover ciertas características y o valores que cualifiquen el existir humano.

Partiendo de que las precarias condiciones de desarrollo social por las que atraviesa el mundo contemporáneo encuentran su razón de ser en las deficientes ideas de "progreso", de "competencia” y de "naturalidad" en ciertos comportamientos sociales y humanos, se vincula a esto el proceso educativo y concretamente la funcionalidad del currículo como una forma que lo ha promovido, pero que también desde su modificación, podría promover otras tendencias con las cuales contribuir realmente a generar comportamientos diferentes en el sujeto que se educa y a través de él, en la sociedad en la que coexiste.

\section{La enseñanza de la literatura en Colombia}

Los lineamientos curriculares de lengua castellana — que son por lo general en los que se incluye lo respectivo a la enseñanza de la literatura- están pensados en relación con el desarrollo de ciertas competencias de carácter comunicativo, en las cuales el estudiante deberá desarrollar una cantidad específica de características que le permite ubicarse dentro de ciertos rangos, en los cuales pueda ser clasificado en relación con el nivel en el que se encuentre, en relación con el objetivo o logro planteado.

No obstante, dichos objetivos están determinados por pruebas de medición - tanto de carácter nacional como internacional - con los que pretenden evaluarse a partir de los procesos de enseñanza-aprendizaje, convirtiéndose entonces en un rasero que califique o descalifique, certificando o no, su competitividad en ámbitos generales que poco o nada tengan que ver con las condiciones inmediatas y reales de su contexto particular.

Ahora bien, pensar en la idea de que la enseñanza de la literatura se intenciona en relación con las características de la ideología que caracteriza una época determinada, demanda una revisión de la historia de la literatura en tanto un método que permita observar dicha posibilidad. En Colombia, un buen inicio sería la historia de la literatura de José María Vergara y Vergara (1974) que da fundamento a la mayor parte de las revisiones y periodizaciones de la literatura durante la primera mitad del siglo XX. Aunque fue una propuesta inconclusa, permitió el planteamiento y caracterización de la noción de "género literario", el cual se convertiría en un referente básico para el diseño del modelo didáctico de las historias literarias con las cuales se enseñaría —en las escuelas y colegios del paísdesde las primeras décadas del XX. 
Ya adentrado el siglo XX, José Joaquín Ortega Torres elabora entre 1934 y 1935 por orden del ministro de Educación Nacional (en ese entonces el escritor Jorge Zalamea, bajo el gobierno de Alfonso López Pumarejo) un programa bandera de reforma educativa, en el que se puso en cuestión la postura y función que tenía la iglesia en la escena política y educativa, al reducir el número de horas de enseñanza de educación religiosa, y a la educación básica y secundaria una libertad que promovía la inclusión de profesores laicos, con lo cual se abrió el espacio para hablar sobre sexualidad y permitir la lectura —en colegios y universidades - de libros de carácter filosófico y liberal, o existencial y crítico como Cuatro años a bordo de mí mismo del escritor colombiano Eduardo Zalamea (mal visto y a veces prohibidos por la iglesia).

Además, bajo este mismo gobierno, se propuso la escuela obligatoria y gratuita, con lo cual se restaba significativamente el poder y ejercicio formativo a la iglesia. Sin embargo, la enseñanza de la literatura se redujo al estudio de los autores con mayor intensidad que en sus obras, siendo esencialmente descriptivo. Esta historia incluye una recopilación de comentarios de críticos e historiadores sobre las obras que son relevantes en las aulas: José Manuel Marroquín, Julio Arboleda y Gregorio Gutiérrez González (Vallejo Murcia, 2006). Relevancia que pareció estar sujeta a las formas específicas, a los intereses y apuestas de carácter ideológico, y a las nociones prefiguradas de desarrollo, determinadas por las formas industrializadas del momento.

En estas circunstancias, el papel que tuvieron las editoriales fue determinante, ya que estas re-editan y reimprimen los libros de texto que ya se utilizaban en la escuela europea desde la segunda mitad del siglo XIX, y en los que se podía percibir los cambios de perspectiva literaria y la relación curricular de la enseñanza de la lengua y la literatura en aras de moldear los comportamientos sociales en esa dirección.

Muchos de estos manuales o textos de estudio, que aparecieron entre 1934 y 1937, subsistieron hasta los años 70 mediante reediciones que - en número muy significativo - se realizaron en la editorial Bedout de Medellín y siempre direccionados por las indicaciones curriculares y didácticas del Ministerio de Educación Nacional.

No se puede pasar por alto que, en 1928, el sacerdote jesuita Félix Restrepo funda la Librería Voluntad, la cual en sus inicios distribuía y vendía libros de texto y literatura universal que importaba desde España, los cuales —en su gran mayoría y de manera inmediata- fueron incluidos en las propuestas curriculares de la época, lo que indica la relevancia del criterio religioso en la selección de textos para ser leídos por los estudiantes colombianos.

Entre 1928 y 1929 se crea otra editorial religiosa, pensada y organizada por la comunidad de las hermanas Paulinas y el Sacerdote Alberione, para la importación y venta de libros de religión, historia sagrada y vidas de santos, con el eslogan preciso y que no necesita más explicación: “[n] uestras librerías no son para hacer dinero, sino para beneficio de la gente. La librería es un Templo; el librero un predicador”. 
Durante la República Liberal (1930-1946) hay una preocupación por la edición y difusión de libros, aunque la mayor parte de la población fuera analfabeta y consumiera publicaciones periódicas (semanarios y revistas), que en la mayoría de los casos no tenían censura; por lo tanto, se permitía dar a conocer textos de pensadores y escritores con los cuales hubiera algún tipo de relación a partir de sus lineamientos políticos e ideológicos, ya que estos inculcarían y redirigían las formas de pensar y actuar de los lectores, y con ello marcar el norte de la vida cultural latinoamericana. Así, por ejemplo, revistas norteamericanas que se publican en español para América Latina, como Life y Time, sin olvidar la muy significativa Selecciones, evidencian un profundo carácter transculturizante de corte neocolonialista.

En Colombia, a mediados del siglo XX, dos revistas: Mito (1955-1962) y Eco (1960-1984) — de acuerdo con Jorge Orlando Melo— se dieron a la tarea de crear lo que existía solo en grado muy pequeño: un público, un sistema de acceso, un espacio cultural creativo no determinado por los procesos de carácter ministerial y estatal; un público que sabiendo leer y escribir, y habiendo pasado por la escuela secundaria o universitaria, potenciara una forma de resistencia a esos intereses gubernamentales, siempre tan comprometidos con los foráneos; sin embargo, el nivel de analfabetismo era muy alto y el acceso a la educación estaba muy restringido por factores de índole económico y, aunque increíble, de carácter ideológico, razón por la que es comprensible que el boom de la secundaria y la universidad se desarrollara tan solo a partir de los años 60.

Durante la segunda mitad del siglo XX aparecen otras editoriales de textos escolares como Norma (en 1960), que inicialmente cubre solamente el mercado nacional, incluyendo la línea de textos literarios; esto implicó que la propuesta de planes de lectura que, además se reducían a simples listados de libros que ellos mismos editaban, se convirtieran en referentes de estudio que poco o nada tenían que ver con el análisis de obras y los contextos histórico, político y social, lo cual habría ido en contrasentido con las caracterizaciones formativas que tenía en su perspectiva la tendencia centralista y dominante de los gobiernos del momento.

En general, puede decirse que la historia del país ha estado discreta e indirecta - pero fuerte y contundentemente- determinada por la relación con los periódicos, las revistas y las editoriales; la publicación y comercialización de libros ha estado muy definida por los intereses personales e intereses partidistas y estatales, lo que a su vez ha incidido bastante en los procesos educativos y culturales de la nación.

De acuerdo con Robert Arvone (1978), durante la dictadura de Gustavo Rojas Pinilla y luego durante los cuatro gobiernos del Frente Nacional, el Estado colombiano utiliza el poder político, económico y militar para anular la acción de los movimientos populares, que en cierta medida se preocupaban por una educación igualmente popular, pero que se da en una sociedad indiscutiblemente clasista y discriminatoria, en la que se hace una diferencia radical entre la escuela pública y la privada; y en la 
que cualquier movimiento de carácter obrero o universitario era ligado, inminente y convenientemente, con la izquierda y la guerrilla.

Esto significó que los contenidos de los currículos — parte esencial de los procesos educativos - fueran pensados y puestos en práctica a partir de las líneas definidas por la burocracia internacional, bajo la supervisión y amparo de las entidades que brindaron el "apoyo" y la asistencia externa; con lo cual, se entorpeció muchísimo más el funcionamiento adecuado, sujeto a realidades consecuentes, del sistema educativo nacional.

Así, al servicio de las clases dominantes, y en menor medida de la clase media, y muchísimo menos marcado a los sectores populares y rurales, los procesos formativos y educativos en Colombia han estado orientados a fortalecer y hacer crecer el poder del sector privado, que presiona o impone políticas educativas convenientes para ese statu quo. La enseñanza de la literatura no está libre de este influjo.

Es con la Ley del libro de 1993, que particularmente se posesionan con mayor fuerza las editoriales extranjeras, en especial las españolas, las cuales presentan los programas curriculares, los libros de texto, de lengua y literatura, con guías que le indican al maestro qué debe saber y hacer, cuáles libros debe leer y cómo debe hacerlo.

Estos programas buscan conservar dentro de lo doctrinal, dejando de lado las posibilidades que ofrece la literatura en la formación del ser humano, a la vez que son eficaces métodos para configurarlo; emplean la enseñanza de la literatura para reproducir los modelos y las conductas, para legitimar comportamientos adecuados a la conservación de esas formas determinantes de los sistemas socio-culturales establecidos, fundamentados en criterios cada vez más banales y frívolos, definidos por los departamentos de mercadeo, a los que se les suma la posición acrítica y silenciosa de la comunidad académica, de la escuela y de las facultades de educación sin opinión y dedicadas a un estudio estructuralista o esteticista de la literatura, donde muchos profesores de literatura — universitarios incluso - utilizan a Homero para hacer categorías gramaticales o para justificarse unos tragos con el juego "si pierde se lo toma”, argumentando que buscan la empatía del estudiante y el desarrollo de nuevas herramientas didácticas y metodológicas.

El final de siglo anterior y el comienzo del actual han estado permeados por una forma de violencia que, aunque consecuencia de las viejas formas de violencia política, han generado nuevas y diferentes formas en las zonas rurales e incluso en los suburbios de las ciudades, afectando drásticamente los procesos educativos e incluso los imaginarios y la visión de mundo de los sujetos que las habitan?.

Ahí el control sobre lo que se lee, pero también la promoción de lo que debería leerse y cómo se ha configurado como modificación sustancial y como aporte

7. Este tema fue abordado en la tesis de la Maestría en Literatura Colombiana: La visión de mundo y su incidencia en los procesos de interpretación y explicación del texto narrativo, que además es un producto directo de esta investigación. 
significativo para los planes de estudio, alterando directamente las formas de realidad y la relación con ella ${ }^{8}$.

A esto se añade que la posibilidad de lectura es controlada de manera sutil, a partir del aumento de precios y la desaparición vertiginosa de las librerías, ante la indiferencia del Estado y sus aparentes políticas defensoras y promovedoras de la cultura del libro, y de los aspavientos para su promoción, en las que los maestros son reclutados para repetir lo que se es dado en los libros de texto, según las orientaciones gubernamentales y de los grupos de poder.

Parece evidente que la censura no es solo a la prohibición, o la orientación reglamentada es más sutil; está incluso en la pobreza de los contenidos especificados en muchos de los libros de texto y la insignificante cantidad de libros ofrecidos por las bibliotecas de las instituciones, a lo que se suma el hecho de que estas generaciones que prefieren la imagen en movimiento, por encima de la interacción con discursos que demandan una actitud y una postura más dialógica, imaginativa y personalizada en el texto escrito, evidencian una serie de características cognitivas y de pensamiento, a las que poco o nada les interesa la más mínima concentración y asimilan —en cambio - fácil y ágilmente los datos relacionados con la banalidad y frivolidad de los objetivos de vida.

Se continúa leyendo literatura clásica como forma de modelización estética, y las formas de enseñanza son los inventarios de autores y obras, y se cuelan lentamente las literaturas que "atontan", como los textos de autoayuda; los textos que pontifican y determinan qué es importante, cómo se debe decir y cómo no pensar; se exprime y se va dejando casi seca la opinión; se enseña literatura, pero son pocos los que en ella encuentran una conexión para la vida.

Ahora, en este momento, durante las dos primeras décadas del presente siglo, no deja de ser preocupante el regreso de varios estados latinoamericanos a la extrema derecha, pues esto prefigura una literatura y una forma de enseñanza, de la misma manera que ya se hizo en Europa y Latinoamérica durante el siglo pasado; tiempos en los que lo social fue solo parte de un eslogan a partir del cual se legitimó una realidad anómala y bastante acomodada a los intereses ideológicos de algunos grupos sociales.

En la misma línea de reflexión y bajo la misma lógica, pensar en la labor del docente implica no la enseñanza de una ciencia sino, la aplicación juiciosa y responsable de leer asertivamente las circunstancias, las características y los componentes de la realidad

8. Bastante significativo y no menos intrigante el hecho de que ciertas obras literarias en Colombia, vinculadas al narcotráfico, al sicariato y en fin a toda esa violencia que se respiró durante los años 80, sean ahora catalogadas como “novelas de identidad nacional”. Véase Valdés León (2010). 
en la que habita como parte de una comunidad y de una totalidad en la cual se hace preciso, más que una incursión cientificista, una con carácter emocional y existencial; más que una reconfiguración de los elementos que constituyen la propuesta pedagógica y didáctica, una actitud que pretenda buscar, analizar y formular los elementos que faltan para dar una orientación en aras de subvertir, alterar o diseñar métodos de enseñanza, que modificando su paradigma, modifiquen la realidad en tanto escenario para el proyecto del ser latinoamericano (Hernández Palencia, 2020).

Una actitud con la cual leer la realidad, no solo de las circunstancias actuales, sino también de las proyecciones consecuentes hacia las cuales quiera llevarse el proceso; es necesario continuar una reflexión dialógica que proponga y abogue por mejores formas del ser y la existencia a partir de una educación diferente, fundamentada en una noción de realidad distinta, una que sea propia, para pensarla con sus formas, circunstancias características, donde la disyuntiva sea el cómo mejorar lo que se es, en concordancia con sus particularidades, y no el cómo mejorar lo que se es, en relación con lo que otras realidades determinan.

\section{Referencias}

Arvone, R. (1978). Políticas educativas durante el frente nacional 1958-1974. Revista Colombiana De Educación, 1(1), 8.37. https://doi.org/10.17227/01203916.4933

Brito, A., y Finocchio, S. (2006). Lenguajes y nuevas tecnologías: Oportunidades y amenazas. IESALC UNESCO.

De Alba, A. (1998). Currículum: crisis, mito y perspectivas. Miño y Dávila editores S.R.L.

De Alba, A. (2007). Clase 20. Los saberes en la relación entre diferencias. Diploma Superior Currículum y prácticas escolares en contexto. FLACSO, Argentina.

Foucault, M. (1994). Hermenéutica del sujeto. La piqueta.

Freud, S. (1930). El malestar en la cultura. Ed. Digital.

Grundy, S. (1998). Producto o Praxis del currículum. Morata.

Hernández Palencia, J. (2020). Aportes para una antropología de la pedagogía latinoamericana. Pacarina del Sur, $11(43)$.

Melo, J. (s. f.). Las revistas literarias en Colombia e Hispanoamérica: una aproximación a su historia. http://www.jorgeorlandomelo.com/bajar/revistas_suplementos_ literarios.pdf 
Kessler, G. (2002). La experiencia escolar fragmentada: estudiantes y docentes en la escuela media en BsAs. UNESCO.

Navarrete Linares, F. (2001). Diálogo con Mijail Bakthine sobre el cronotopo. En La tortuga marina: historia en extinción.

Valdés León, C. (2010). Rosario Tijeras: Construcción identitaria en medio del caos. Informe final de Seminario para optar al título de Licenciado en Lengua y Literatura Hispánica, mención literatura. Universidad de Santiago de Chile.

Vallejo Murcia, O. (2006). El papel de la historia literaria en la enseñanza de la literatura. Lingiística y Literatura, 49, 177-191.

Vergara y Vergara, J. (1974). Historia de la Literatura Colombiana. Fondo de Promoción de la Lectura del Banco Popular. 
